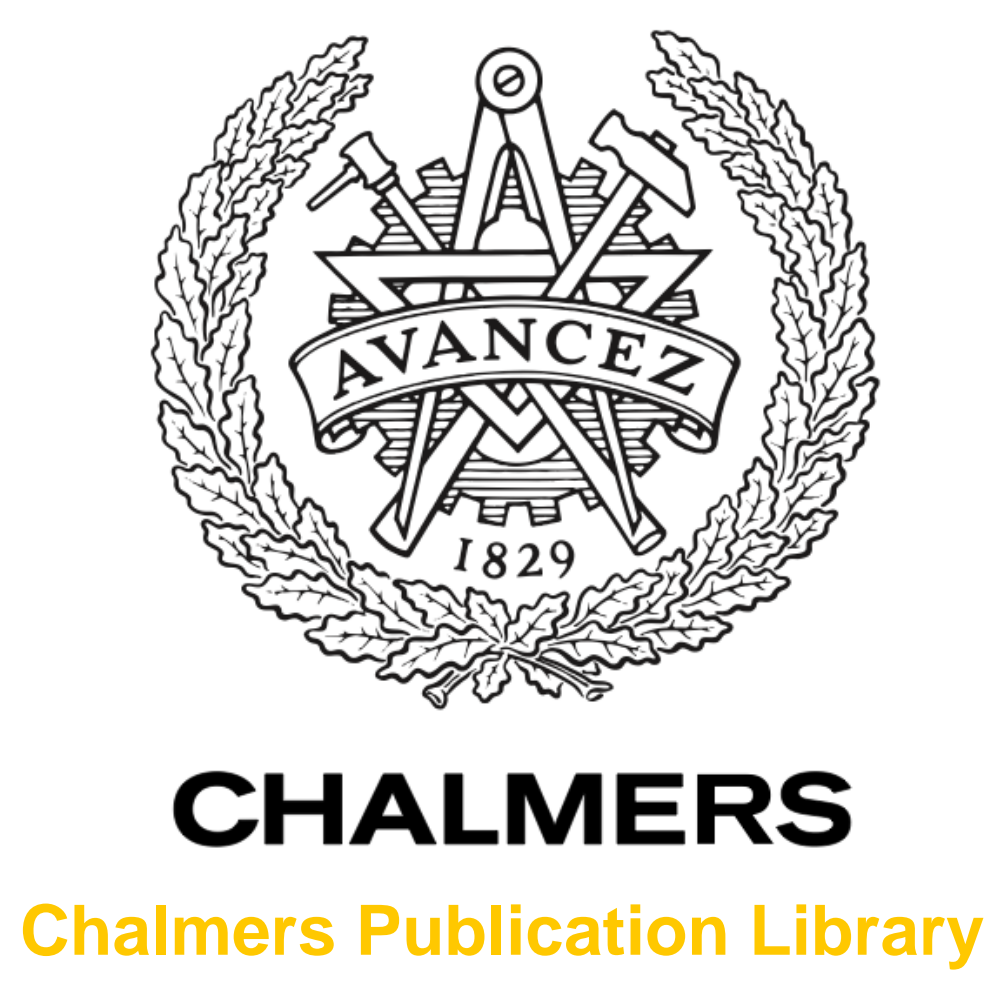

\title{
On the relations between historical epistemology and students' conceptual developments in mathematics
}

This document has been downloaded from Chalmers Publication Library (CPL). It is the author's version of a work that was accepted for publication in:

Educational Studies in Mathematics (ISSN: 0013-1954)

Citation for the published paper:

Bråting, K. ; Pejlare, J. (2015) "On the relations between historical epistemology and students' conceptual developments in mathematics". Educational Studies in Mathematics, vol. 89(2), pp. 251-265.

http://dx.doi.org/10.1007/s10649-015-9600-8

Downloaded from: http://publications.lib.chalmers.se/publication/226683

Notice: Changes introduced as a result of publishing processes such as copy-editing and formatting may not be reflected in this document. For a definitive version of this work, please refer to the published source. Please note that access to the published version might require a subscription. 


\title{
On the relations between historical epistemology and students' conceptual developments in mathematics
}

\author{
Kajsa Bråting $\cdot$ Johanna Pejlare
}

Published online: 27 March 2015

(C) Springer Science+Business Media Dordrecht 2015

\begin{abstract}
There is an ongoing discussion within the research field of mathematics education regarding the utilization of the history of mathematics within mathematics education. In this paper we consider problems that may emerge when the historical epistemology of mathematics is paralleled to students' conceptual developments in mathematics. We problematize this attempt to link the two fields on the basis of Grattan-Guinness' distinction between "history" and "heritage". We argue that when parallelism claims are made, history and heritage are often mixed up, which is problematic since historical mathematical definitions must be interpreted in its proper historical context and conceptual framework. Furthermore, we argue that cultural and local ideas vary at different time periods, influencing conceptual developments in different directions regardless of whether historical or individual developments are considered, and thus it may be problematic to uncritically assume a platonic perspective. Also, we have to take into consideration that an average student of today and great mathematicians of the past are at different cognitive levels.
\end{abstract}

Keywords History of mathematics · Mathematics education - Epistemology of mathematics $\cdot$ Recapitulation · History and heritage

K. Bråting

Department of Education, Uppsala University, Uppsala, Sweden

e-mail: kajsa.brating@edu.uu.se

J. Pejlare

School of Education and Behavioural Sciences, University of Borås, Borås, Sweden

e-mail: johanna.pejlare@hb.se

Present Address:

J. Pejlare $(\triangle)$

Department of Mathematical Sciences, University of Gothenburg and Chalmers University of Technology, Göteborg, Sweden

e-mail: johanna.pejlare@gu.se 


\section{Introduction}

During recent decades there has been an increase of research in the history of mathematics, which seems to have influenced other domains as well. Mathematics syllabuses at schools and universities often refer to the history of mathematics, and research in mathematics education more frequently involves history of mathematics. A sufficient comprehensive description of the multifarious role of the history of mathematics in mathematics education is given in Tzanakis and Arcavi (2000). The history of mathematics is utilized in the research field of mathematics education in different ways, such as in the formation of theoretical frameworks (Jankvist, 2009; Jankvist \& Kjeldsen, 2011; Sfard, 1991, 1995), as teaching and learning via primary historical sources (Fenaroli, Furinghetti, \& Somaglia, 2014; Glaubitz, 2011; Jahnke, 2000; Pengelley, 2011, 2012), as a motivating factor in the learning of mathematics (Farmaki \& Paschos, 2007), as a means to device educational material and implement a teaching approach (Jankvist, 2011; Panagiotou, 2011; Siu, 2000), and as evolutionary arguments (Piaget \& Garcia, 1989). Difficulties in the effort to combine history of mathematics and mathematics education has also been considered. For example, Fried $(2001,2007)$ points out that the commitment to teaching modern mathematical techniques is at odds with the historian's commitment to avoid anachronism and to understand the mathematics of the past as something more than an older version of modern mathematics.

The evolutionary arguments, or the genetic approach, originates in the German biologist Ernst Haeckel's (1834-1919) law of biological development, wherein he claims that "ontogeny recapitulates phylogeny". Although Haeckel's theory of recapitulation is now discredited among biologists, it had a strong influence on social theories and educational practices developed during the end of the 19th century. According to the psychological version of the theory of recapitulation, it is assumed that the present intellectual developments are to some extent a condensed version of those of the past. This would imply that while developing the understanding of, for example, a mathematical concept, the student recapitulates the historical development of the concept. As further outlined in Fauvel (1991), Furunghetti and Radford (2002, 2008), Mosvold (2003) and Schubring (2011), the theory of recapitulation was adopted among mathematicians and mathematics educators in the late 19th century. For example, Henri Poincare (1854-1912) suggested that "the educators' task is to make the children follow the path that was followed by their fathers" (Poincaré, 1899, p. 159). Also Felix Klein (1849-1925) had an interest in mathematics education, which he believed was closely connected to the history of mathematics. He was convinced that the road to discovery, rather than the formal arguments, was of most importance. Furthermore, Otto Toeplitz (1881-1940) pleaded for a genetic methodology in the teaching of mathematics, using history as a didactical means. In particular he considered the role of teachers, understanding teachers as actively reflecting the history of mathemaics and transmitting its essence to the students (Schubring, 2011).

As described in, for example, Furunghetti and Radford (2002, 2008) and Radford (2000), during the last century there was a growing interest in the relation between ontogenesis and phylogenesis among psychologists. For example, Lev Vygotsky (1896-1934) dealt with the problem of recapitulation, emphasizing the epistemological role of culture. He pointed out that the activity of mental functions is modified by the use of tools and artefacts, such as,

\footnotetext{
1 "La tâche de l'éducateur est de faire reprasser l'esprit de l'enfant par où a passé celui de ses pères."
} 
for example, clay tables, counting boards, words and language. Thus, since the tools differ among different cultures, and sociohistorical conditions vary among different historical periods, recapitulation is invalidated. Jean Piaget (1896-1980) and Rolando Garcia (19192012) favoured a parallel between historical and psychological developments (Piaget \& Garcia, 1989). They disputed Haeckel's recapitulation, suggesting that this parallel must be seen not in terms of content but in terms of invariable mechanisms for allowing the acquisition of knowledge (Furinghetti \& Radford, 2008). They tried to show that the mechanisms of passage from one historical period to the next are analogous to those of the passage from one psychogenetic stage to the next, and thus these mechanisms cannot be modified by, for example, culture.

In this paper we discuss studies in mathematics education where evolutionary arguments have been invoked erroneously. In particular, we argue that there are three essential problems that may arise when historical epistemology of mathematics is paralleled to students' conceptual developments in mathematics: the problem of not considering different conceptual frameworks, the problem of tacitly assuming a platonic perspective, and the problem of not considering differencies in cognitive levels. For example, we argue that there are studies where historical evolution is compared to students' conceptual developments which are based on a belief that the concepts used in schools and at universities today are the "correct" definitions that mathematicians have strived for throughout history. That is, one can say that these studies tacitly assume a platonic perspective of mathematics or, as we are about to describe, utilize a typical heritage approach (in terms of Grattan-Guinness' distinction between history and heritage (Grattan-Guinness, 2004a, b). Furthermore, we argue that it is problematic when the historical evolution is paralleled to students' conceptual developments since the focus is often restricted to only one specific concept and different conceptual frameworks from different periods of times and from different cultures are not taken into consideration. For instance, it is questionable to claim that students today understand the function concept in a similar way as, for example, Leonhard Euler (1707-1783) did during the 18th century, since Euler not only was at a different cognitive level compared to today's students, but also based his function concept on a different conceptual framework compared to the students.

\section{History versus heritage of mathematics}

During recent years the legitimacy of historical research in mathematics has been debated. One crucial question has concerned how historical texts in mathematics should be interpreted in a proper (and historically correct) way. Grattan-Guinness (2004a, b) has considered this issue on the basis of the two approaches:

- $\quad$ the history approach, versus

- the heritage approach.

Although the distinction between history and heritage has mainly been considered within the community of history of mathematics, its relevance for mathematics education has also been discussed. Grattan-Guinness (2004a) points out that mathematics education can profit from both history and heritage, which has been discussed by Rogers (2010) and Tzanakis and Thomaidis $(2011,2012)$. In this paper the distincion between history and heritage will be used in connection with our problematization of didactical studies where historical epistemology is related to students' conceptual understanding in mathematics. Let us first give a brief description of Grattan-Guinness' distinction. 
History deals with what happened in the past, regardless of the modern situation. In order to study a specific mathematical theory, definition, theorem, concept etc., the history approach focuses on the details of its original development, its prehistory, the chronology of progress and its impact in the years immediately following. Grattan-Guinness (2004a, b) argues that history addresses the question "what happened in the past?" but also the question "what did not happen in the past?" In order to answer the corresponding questions "why?" it gives descriptions and also attempts explanations. The history approach may also consider differences between the historical notion and more modern notions that are seemingly similar. A concrete example of interpreting historical texts with the history approach is the following: If Euler's function concept is studied the focus would be to analyse his function concept on the basis of the mathematical context at that particular time period, without being influenced by the modern function concept. The fact that the function concept during the 18th century was quite different compared to today would not be a main point.

Heritage refers to the impact of a certain mathematical notion (a mathematical theory, definition, theorem, concept, etc.) upon later work. The main focus is usually the modern form of the notion studied, with attention paid to the course of its development and when appropriate the modern notions are inserted into the notion studied. Grattan-Guinness summarizes: "Heritage addresses the question 'how did we get here?' and often the answer reads like 'the royal road to me'" (Grattan-Guinness, 2004b, p. 165).

One should keep in mind that the utilization of the heritage approach often results in a modernization of old results in order to show their current place in mathematics, but the historical context is not always taken into account. Grattan-Guinness (2004b, p. 163) points out that this is perfectly legitimate, as long as the heritage approach is not mixed up with the history approach. That is, it is important to take into account that mathematicians of the past based their definitions of the conceptual framework available at the time and to not assume that they necessarily strived for the modern definitions of today. An example of mixing up history with heritage is to claim that Euclid was a "geometric algebraist", in the sense that he was handling geometrical notions but he actually was practising common algebra. ${ }^{2}$ However, to insert modern notions of algebra into Euclid's work is perfectly legitimate and would be a typical example of using the heritage approach. The problem occurs if one claims that algebra is discovered in Euclid's work. ${ }^{3}$

\section{Problems arising from posing parallelism claims}

In order to describe and explain students' conceptual developments in mathematics, evolutionary arguments have become a topic largely shared within mathematics education. For example, bearing on the ideas of "developmental invariants" initiated by Piaget, Sfard $(1991,1995)$ has developed a theoretical framework based on the belief of a parallel between the historical development of mathematical concepts and the development of students' understanding of the concept. In particular, she claims that "difficulties experienced by an individual learner at different stages of knowledge formation may be quite close to those that once challenged generations of mathematicians" (Sfard, 1995, pp. 15-16). In empirical studies aiming at comparing students' learning of mathematical concepts with the historical

\footnotetext{
${ }^{2}$ A well-known debate regarding this issue can be found in Unguru (1975) and Weil (1978).

${ }^{3}$ Discussions regarding modern interpretations of Greek mathematics can be found in Grattan-Guinness (2004a, b).
} 
development of the same concept, the conclusion is often made that students' conceptual understanding corresponds to a specific time period in the historical development of the concept. Another conclusion that is often made is that students learn a specific concept in tandem with the historical development of that concept (see, for instance, Juter (2006) and Moreno and Waldegg (1991)). In this section we elucidate, in turn, three essential problems that may arise in these studies with reference to evolutionary arguments: the problem of not considering different conceptual frameworks, the problem of tacitly assuming a platonic perspective, and the problem of not considering differencies in cognitive levels.

\subsection{The problem of not considering different conceptual frameworks}

One problem when the historical development of a mathematical concept is compared with students' understanding of the same concept is that concepts from different time periods are based on different conceptual frameworks. For instance, if university students' understanding of the limit concept is considered, it is important to take into account that these students are learning the modern limit concept which is based on the modern concepts of variable, function, continuity, et cetera. That is, the modern limit concept is included in our modern conceptual framework. In the same way, for instance, Euler's idea of infinitesimal quantities is included in the conceptual framework of his time. The point here is that when we compare a specific mathematical concept from one time period with the corresponding concept from another time period, it is necessary to take into consideration that the concept is included in a conceptual framework that differs between one time period and another. This is one reason why it becomes difficult to accept Moreno and Waldegg when they in their study regarding students' understanding of the concept of infinity claim that "the student response schemes are similar to the different response schemes given by mathematicians throughout the history of mathematics" (Moreno \& Waldegg, 1991, p. 211).

The problem of not considering different conceptual frameworks through the history of mathematics may also be an issue as we consider Sfard's theoretical framework. When she undertakes an analysis of mathematical definitions and representations from historical and psychological perspectives, she concludes that many mathematical concepts can be formulated in two fundamentally different ways: operationally (as processes and algorithms) and structurally (as abstract objects) (Sfard, 1991, p. 4). For example, we can consider a function operationally as a certain computational process as well as structurally as a set of ordered pairs. Sfard argues that operational and structural conceptions are complementary, or "opposite sides of the same coin" and that the ability of seeing a concept as both a process and an object is indispensable in order to gain a deep understanding of the concept (Sfard, 1995, p. 37). The transition from computational operations to abstract objects, also known as reification, is defined as an "ontological shift" and is, according to Sfard, a long and difficult process in the historical development as well as in the individual's concept formation.

We agree with Sfard that there may be a tendency in psychological development to move from an operational approach to a structural one. However, we are not convinced regarding claims of parallelism in Sfard's (1991, 1995) papers. Let us, for example, consider the function concept from a historical point of view. In 1748 Euler defined the function as an analytical expression: 4 "A function of a variable quantity is an analytic expression composed in any way whatsoever of the variable quantity and numbers or constant quantities"

\footnotetext{
4"Functio quantitatis variabilis est expressio analytica quomodocunque composita ex illa quantitate variabili et numeris seu quantitatibus constantibus."
} 
(Euler, 1748, p.18). The analytic expression of Euler was, according to Sfard, highly connected to algebraic processes and could thus be interpreted as operational (Sfard, 1991, pp. 14-15). However, it is not clear in what context the interpretation is made. If we consider this definition in terms of Grattan-Guinness' heritage approach, we can interpret it as an operational understanding of the modern function concept. But this should not be mixed up with the history approach: Euler in fact dealt with a very different function concept than the modern one. He neither had access to the modern function concept nor did he struggle to formulate it. He formulated a definition of a function within his conceptual framework which was suitable for dealing with problems in mathematics of that time. After a long controversy with Jean le Rond d'Alembert (1717-1783) regarding the nature of functions to be allowed in problems regarding the vibrating string Euler in 1755 published his highly influential book Institutiones calculi differentialis. There he defined a function in an entirely general way: ${ }^{5}$

If some quantities so depend on other quantities that if the latter are changed the former undergoes change, then the former quantities are called functions of the latter. This denomination is of broadest nature and comprises every method by means of which one quantity could be determined by others. If, therefore, $\mathrm{x}$ denotes a variable quantity, then all quantities which depend upon $\mathrm{x}$ in any way or are determined by it are called functions of it (Euler, 1755, p. 4).

Sfard (1991, p. 15) claims that the operational flavor emanates even more clearly from this definition than from the 1748 version.

Moreover, in studies where students' conceptual understanding are compared with the historical development the focus is often restricted to only one specific concept and other concepts have not been taken into account. We argue that since one concept is included in a whole conceptual framework (which differs from time to time) it is important, from a historical perspective, to consider the concept in view of its proper historical context. In the following we will discuss two examples where students' understanding of the function concept have been studied. In the first example, Kjeldsen and Petersen (2014) are aiming to bridge the history of the function concept with theories from mathematics education. They discuss an implementation of an experimental course in the history of the concept of a function in a Danish high school mathematics classroom. Two conclusions drawn are that some of the participated students "acted according to meta-discursive rules that coincide with Euler's from the 1700s" and that the obstacles created for the students by reading a text by Dirichlet from 1837 can be referred to "differences in meta-discursive rules" (Kjeldsen \& Petersen, 2014, p. 43). In the second example, Viirman, Attorps, and Tossavainen (2010) compare students' definitions of a function with Euler's definition from 1755. One of the conclusions is that "the definitions given by the students mostly resemble an 18th or 19th century view of functions" (Viirman et al., 2010, p. 5).

In both of these examples students' understanding of the function concept has been compared with Euler's definitions. We argue that such a comparison is difficult to make since the students' definitions are based on our modern conceptual framework which differs from the conceptual frameworks from the 18th and the 19th centuries. For instance, the function concept depends on the variable concept and today's variable concept differs significantly from the variable concept that Euler had at his disposal. Domingues (2004) points out that

\footnotetext{
5 "Quae autem quantitates hoc modo ab aliis pendent, ut his mutatis etiam ipsae mutationes subeant, eae harum functiones appellari solent; quae denominatio latissime patet atque omnes modos, quibus una quantitas per alias determinari potest, in se complectitur. Si igitur x denotet quantitatem variabilem, omnes quantitates, quae utcunque $a b \mathrm{x}$ pendent seu per eam determinantur, eius functiones vocantur.'
} 
most mathematicians during the 18th century regarded variables as being quantities that vary, that change in magnitude. For instance, the mathematician Guillaume de l'Hospital (1661-1704) defined variables in the follonging way: "We call variable quantities those that increase or decrease continuously; and to the contrary we call constant quantities those that remain the same while the others change" (l'Hospital, 1696, p. 1). L'Hospital's definition is of course quite different compared to the modern variable concept in the sense that today a variable is viewed as a symbol representing an arbitrary element of a specific set. Even though it is questionable whether students of today understand, for example, the variable concept in its modern form, we believe that there is a point in emphasizing the difficulty of investigating students' understanding of one single mathematical concept without taking the proper conceptual framework into account.

\subsection{The problem of tacitly assuming a platonic perspective}

Let us now consider the second essential problem with studies where students' conceptual development in mathematics is compared with the historical development of the corresponding concept. It seems that these studies sometimes have a tendency to view our modern definitions of mathematical concepts as the final "goals" that mathematicians in the past were striving for. That is, the mathematical definitions that we teach at university courses today are viewed as the "correct" definitions that mathematicians finally have discovered, so to speak. Perhaps one can say that studies of this kind presuppose a platonic view of mathematics. One such example is when Juter in a study of students' understanding of the limit concept claims that the students "paralleled the historical development of the concept in the sense that they were able to solve tasks in the beginning before they could explain the theory but that with time the theory became clear to them" (Juter, 2006, p. 426). A platonic perspective of mathematics is even more evident when she claims that historically limits were first treated in problem solving situations, but "with time and through many mathematicians' efforts, the theory became clear" (Juter, 2006, p. 426). We may argue that Juter in her study uses a heritage approach (in terms of Grattan-Guinness), considering the historical development as "the royal road to me", but this becomes problematic as it is mixed up with a history approach.

From a historical point of view though, as also argued in for example Bagni (2004) and Radford (1997), it seems unlikely that mathematicians of the past were struggling to bring their concepts as close as possible to our modern ones. For example, there are alternative ways of defining mathematical concepts. As we will consider further in Section 4.2, in non-standard analysis, which is an alternative to our standard analysis, the limit concept is based on infinitesimals which give a consistent theory within non-standard analysis. Another problem with Juter's quotation above is that it is difficult to claim that "the students parallelled the historical development of the limit concept", since the historical development of a mathematical concept has not been necessarily unique, that is, there is not necessarily one royal road to the modern definition. In Section 4.1 we exemplify this with the historical development of negative numbers which is different in western Europe compared to that in China.

\footnotetext{
6“On appelle quantités variables celles qui augmentent ou diminuent continuellement; \& au contraire quanitités constantes celles qui demeurent les mêmes pendant que les autres changent."
} 
Platonism involves the idea of an ideal perfect structure "out there" for us to discover. A platonic perspective may of course be an acceptable perspective espoused by teachers, shaping the way of teaching and letting the students rediscover mathematics. However, when history of mathematics is considered, a "correct definition" should be referred to as a correct definition in a specified conceptual or mathematical framework. In mathematics and its history it is very common to define a concept in many different ways (for example, irrational numbers as equivalence classes of rational number sequences, or as Dedekind cuts) or using different definitions to describe the same phenomenon (for example Isaac Newton's (1642-1727) fluxions and Gottfried Wilhelm von Leibniz' (1646-1716) infinitesimals to describe the rate of change of a quantity). Therefore, we argue that a platonic perspective is problematic when we consider the history of mathematics.

Radford argues that the history of mathematics often is presented as episodic narratives implicitly underlain by an apriorist platonic epistemology (Radford, 1997, p. 26). Typically, the aim is to show students how past mathematicians succeeded in discovering mathematical concepts. We can perhaps see traces of this belief in Sfard (1991). It seems that she, in her historical investigation of the function concept, does not take the historical context into proper account when she, for example, claims that the problem with early definitions of the function concept is that they leaned on the concept of variable which was "rather fuzzy and escaped every attempt at reification" (Sfard, 1991, pp. 14-15). Schubring criticizes Sfard for understanding the history of mathematics as a "ready-made, unquestionable product suited to confirm her claims about historical and psychological parallels" (Schubring, 2011, p. 85). We agree with Schubring that Sfard's description of the history of mathematics is simplified, at least from a history approach in terms of Grattan-Guinness. But perhaps we could say that she utilizes a heritage approach when interpreting the history of mathematics, focusing on the modern form of the concepts.

\subsection{The problem of not considering differencies in cognitive levels}

The third essential problem with studies where students' conceptual developments are compared with the historical development is what we refer to as the problem of not considering differencies in cognitive levels. Let us consider one of Juter's (2006) conclusions in the study referred to above, where she claims that "the students with problems explaining the limit definition appeared to be mainly at the stage of Newton and Leibniz with a sense of what they do but lacking the means to strictly formulate and explain the concept of limits" (Juter, 2006, p. 427). ${ }^{7}$ It seems unfair to compare a student of today (just about to learn the fundamental concepts of calculus) with Newton, Leibniz, or Euler. Not only do they consider different definitions in different mathematical frameworks; we also have to assume that the students are at different cognitive levels compared to famous mathematicians of the past. Even if Euler, according to Sfard's definition of reification, may not have reached the stage of a reified understanding of his definition of a function, since he remained partially linked to an operational frame, he most probably was at a higher cognitive level compared to the average student of today, who struggles to understand the modern definition of a function but still considers it as an analytical expression, and being far from the reified stage.

\footnotetext{
${ }^{7}$ This conclusion is similar to the conclusion of Viirman et al. (2010, p. 5) that we referred to above: "the definitions given by the students mostly resemble an 18th or 19th century view of functions".
} 
Moreover, the students today have seen the definitions of the concepts that they are about to learn and are trying to handle these concepts "from above". The students' mistakes probably depend on misunderstandings of the definitions, or arithmetical miscalculations. Historical mathematicians, on the other hand, were developing the mathematical concepts "from below": they carefully chose the best ways to reach their goal and they in most cases used the defined concepts correctly. If it later turned out that their definition was not appropriate then they corrected or reformulated it.

Kjeldsen and Petersen (2014) make a similar conclusion as Juter in their study regarding the history of the function concept and students' conceptual formation of functions. They claim that "the experiment revealed that many of the students have a concept image that was in accordance with Euler's rather than with our modern concept definition and that they have process oriented thinking about functions" (Kjeldsen \& Petersen, 2014, p. 29). We believe that it is problematic to claim that many of the students' concept image were in accordance with Euler's definition, especially in the light of how Tall and Vinner define "concept image" as "the total cognitive structure that is associated with the concept, which includes all the mental pictures and associated properties and processes" (Tall \& Vinner, 1981, p. 152). As already mentioned, one cannot assume that students are at the same cognitive level as famous mathematicians of the past.

However, we agree with Kjeldsen and Petersen that students often have a processoriented thinking about functions, and there is a point in questioning whether students of today really understand the function concept in its modern form. But this does not imply that we unquestionably can draw conclusions from comparing students' conceptual understanding with those of famous mathematicians of the past. Nevertheless, we may agree that one can perhaps trace the parallelism suggested by Sfard if we consider the historical development of mathematical concepts with a heritage approach without taking the historical context into consideration. Then we possibly could compare the students' understanding of a modern concept with a modern interpretation of a historical definition of the concept. This may, of course, according to Grattan-Guinness (2004b, p. 163) be completely legitimate, as long as we do not mix up the heritage approach with what actually happened in the past. As historians of mathematics, however, we question what the point of this would be.

\section{Alternative historical developments and alternative theoretical frameworks}

In order to further justify our claims we will in this section consider two examples. In the first example we consider the historical development of the understanding of negative numbers. We emphasize the epistemological role of culture in the historical development of mathematical concepts, arguing that there is not a foregone conclusion about how a mathematical concept has developed. In the second example we consider Schmieden and Laugwitz' (1958) version of non-standard analysis, which is an alternative theory to our ordinary analysis. We argue that there are different ways of defining mathematical concepts which also lead to consistent mathematical theories. Thus it may be problematic to uncritically take a platonic view of mathematics.

\subsection{Negative numbers and the role of culture}

In this section we argue that local and cultural ideas about science and mathematics influence the development in different directions. We find one example of this when we study 
the development of the notion of negative numbers in China and in western Europe. References to negative numbers were first made in China in Jiuzhang Suanshu (Nine Chapters on the Mathematical Art); for an overview of the text see Lam (1994). This is a practical handbook of mathematics containing mathematical concepts and methods, and it played a fundamental role in the development of Chinese mathematics. Liu Hui wrote a commentary on Nine Chapters in 263 AD, but the mathematics described had been developed from about 1000 BC. Negative numbers are introduced and used in Chapter 8 , where a method is given for solving systems of linear equations. The method is very similar to Gaussian elimination: the coefficients are placed in a rectangular array, or matrix, of rod numerals and thereafter reduced to triangular form. In this algorithm negative numbers appear.

Arithmetic in China was carried out using counting rods from about the first millenium BC (for a full treatment, see Lam \& Ang, 2004; Lam \& Shen, 1989). The rods were arranged in rows using a decimal place notation, and when performing calculations different numbers were laid out in a series of rows forming a grid. Counting rods with different colours were used to symbolize subtraction: a black number to be subtracted from a red one. As the red counting rods represented positive numbers it was not hard to give the black counting rods a meaning as negative numbers. Negatives arose naturally in solving concrete problems, and in Liu's commentary rules for adding and subtracting, positive and negative numbers were explained.

In western Europe, however, the concept of negative numbers was resisted for a long time. Knowledge of negative numbers and algebraic techniques had reached western Europe in the 13th century. For example, negative numbers appear in many problems in Liber abici (1202/1228) by Leonardo of Pisa (Fibonacci, 1170-1250). He generally rejected negative solutions but reformulated the problems when possible to permit a solution. In problems concerning money he interpreted negatives as debts, borrowed money or invested capital. However, when a reinterpretation was impossible, the negative solution was rejected as inconvenience (Schubring, 2005, p. 39).

In Ars Magna (The Great Art) from 1545 Girolamo Cardano (1501-1576) gave methods of solution of the cubic and quartic equations. He rejected negative numbers, and since all the coefficients then had to be positive he had to describe how to solve 13 distinct cases of cubic equations. He did recognize that some of his equations had negative solutions, but he systematically ignored them. In the chapter On the rule for postulating a negative he considers problems with complex roots which he calculates. However, he saw no physical meaning to it and stated "so progresses arithmetic subtlety, the end of which, as is said, is as refined as it is useless" (Cardano, 1545/1993, p. 220).

Negative numbers started being used systematically not only through the problem of applying algebraic syntactical rules to the theory of equations, but also through the problem of dealing with the correspondence between the terms of an arithmetical and a geometrical progression (Thomaidis, 1993; Thomaidis \& Tzanakis, 2007). In his book Arithmetica Integra (1544) Michael Stifel (1486-1567 or 1487-1567) examined the correspondence between the two progressions, in fact being a logarithmic system of base 2, noting that he could extend the correspondence to negatives of the arithmetical and fractions of the geometrical progression (Panagiotou, 2011, p. 5). He clearly stated that the negative numbers are less than zero. However, he did not acknowledge equal status for positive and negative numbers, declaring positive numbers to be real numbers and negative numbers were ascribed only an imagined existence (Schubring, 2005, pp. 40-41). 
Important work on negative numbers should be attributed to John Wallis and Isaac Newton in the 17th century (Schubring, 2005, pp. 89-92). For example, in Treatise of Algebra from 1685, Wallis gives a simple and clear definition of positive and negative numbers as contrary signification:

If + signify Upward, Forward, Gain, Increase, Above, Before, Addition, \& c. then - is to be interpreted of Downward, Backward, Loss, Decrease, Below, Behind, Subduction, \& c. And if + be understood of these, then - is to be interpreted of the contrary (Wallis, 1685, p. 16).

Mumford (2010, p. 140) argues that the European resistance to negative numbers was due to two factors. The first factor is Euclid, whose Elements for generations came to define what is and what is not mathematics. In the Elements Euclid deals with magnitudes and ratios, and these concepts do not provide numbers that can be negative or even zero. The second factor is the relationship between negative numbers and imaginary numbers: at the same time as negative numbers should have been accepted, imaginary numbers also cropped up. There was a problem with square roots because of the negatives, and thus it may have seemed most suitable not to consider the negatives at all.

The historical development of the notion of negative numbers in different cultures illustrates clearly that there are differences among local and cultural ideas about science and mathematics, and their objects and methods, that influence this development. In China the number rod system had been developed and its availability made it natural to simply use a different colour to represent, for example, debts. However, in Europe different colours were not used to represent positives and negatives. Instead the Greek heritage of magnitudes and ratios in combination with the problem of accepting imaginary numbers for a long time kept back the full understanding and acceptance of negatives.

\subsection{Non-standard analysis}

In this section we exemplify that a mathematical phenomenon can (rigorously) be defined in different ways depending on its theoretical framework. For instance, Newton's fluxions and Leibniz' infinitesimals both describe the rate of change of a quantity. Complex numbers can be described as ordered pairs of real numbers, or as vectors, or as rotations and dilatations in the plane, or as the algebraic extension of the algebraic field of reals by $i=\sqrt{-1}$. One description is just as good as the other. In the following example we will further point out that the definitions we use in mathematics courses at universities today are not necessarily the "best" definitions that mathematicians in the past were striving for. In our example we point out that in non-standard analysis it is possible to formulate an even better version of the well-known Cauchy's sum theorem compared to the formulation that we use in our standard analysis today.

Non-standard analysis is an alternative theory to our ordinary analysis used in university courses in mathematics today. The classical non-standard analysis is associated with Robinson (1996), but there is also a more intuitive alternative, the so called $\Omega$-calculus, developed by Schmieden and Laugwitz (1958); our example refers to the latter.

The $\Omega$-calculus deals with sequences of real numbers, that is, functions from the natural numbers to the reals. Schmieden and Laugwitz introduce $\mathbf{R}^{\star}$ as the set of all infinite sequences $\left(x_{1}, x_{2}, x_{3}, \ldots\right)$, where each term $x_{k}$ is a real number. Furthermore, an infinitesimal quantity is defined as a sequence having zero as a limit, for instance $\frac{1}{n}=\left(1, \frac{1}{2}, \frac{1}{3}, \frac{1}{4}, \ldots\right)$. 
The modern version of Cauchy's sum theorem in standard analysis is the following: If a sequence of real valued continuous functions, $f_{n}$, converges uniformly to a function $f$, then $f$ is a continuous function. In this case uniform convergence is a sufficient but not a necessary condition for the theorem to be true. In non-standard analysis it is possible to establish Cauchy's sum theorem utilizing a weaker convergence condition than uniform convergence, yet sufficient for the theorem to be true (Palmgren, 2007, pp. 171-172). In fact, Palmgren proves that this weaker convergence condition not only is sufficient, but also a necessary condition for the theorem to be true (Palmgren, 2007, p. 171). That is, one obtains a more precise version of Cauchy's sum theorem in non-standard analysis than in our modern standard analysis.

Our conclusion here is that there are alternative ways of defining mathematical concepts within other theoretical frameworks than our standard analysis. In our example above we point out that there exists even "better" descriptions of mathematical phenomena than in our ordinary standard analysis. This implies that it is problematic to draw the conclusion that students' conceptual development follow the historical development of the same concept, since that would presuppose that the definition we use today is the "correct" one, so to speak.

\section{Concluding remarks}

In this article we have revealed some pitfalls of using history of mathematics in mathematics education because it may lead to a confusion among researchers, teachers and students. We have considered some examples of research in mathematics education where students' conceptual developments in mathematics have been compared to the historical epistemology of mathematics. We argue that it is problematic to expect to find a historical equivalent to this development when students are still struggling to master a mathematical concept. The historical development of mathematics, considered in its proper context, is not uniform throughout the world although it is often implicitly understood as such. As we have seen from the example with negative numbers, the development of one concept can be different in different parts of the world and evolve during different time periods, depending on the development of cultural ideas and the needs of society. The concepts we use today are also not the only possible "correct" concepts, even though they may be considered as such from a platonic perspective. We have seen that, for example, standard analysis could be replaced by non-standard analysis where concepts such as continuity and convergence are formulated in a different manner. This example illustrates that there are different possible conceptual frameworks that can be used when formulating the same phenomenon. Furthermore, we argue that it is unfair to draw the conclusion that a student who has still not understood, for example, the limit concept and still struggles when trying to use it, has reached the level of, for example, Newton's or Leibniz's understanding of the concept. However, mathematicians in the history often worked with sensory images and for a long time mathematics was thought about as something sensory and concrete, which maybe also many students in some sense do today. Nevertheless, it is optimistic to compare students' conceptual developments with history and assume that students are rational in their conceptual developments. At least we must assume that an average student IS at a significantly lower cognitive level than the great historical mathematicians. In fact students are making mistakes when they try to use a mathematical concept they still do not master and it is difficult to draw conclusions on the basis of these mistakes. 
While the aim of this article has not been to discusss the usage of history of mathematics in teaching contexts as such, we do hope that this article will be useful for researchers and teachers that might be too enthusiastic proponents of integrating history in mathematics education so that they tend to ignore the subtleties inherent. We would like to point out that using history of mathematics can play an important role in the teaching and learning of mathematics. We side with Grattan-Guinness that mathematics education can profit from both history and heritage, as long as the two approaches are not mixed up. Heritage is utilized frequently, as for example when Euclid's Elements is used in the teaching of algebra. But we also have to pay attention to the history approach and its merits in thinking about mathematics as cultural inheritance. We believe that the knowledge of history of mathematics is very useful to understand and appreciate the mathematics of today.

Acknowledgements We would like to thank the anonymous referees with the help of whom the manuscript has improved considerably.

\section{References}

Bagni, G. T. (2004). Exhaustion argument and limit concept in the history of mathematics: Educational reflections. In F. Furinghetti, S. Kaiser, \& A. Vretblad (Eds.), Proceedings of History and Pedagogy of Mathematics 2004, Uppsala (pp. 94-103). Uppsala, Sweden: University of Uppsala.

Cardano, G. (1993). Ars Magna or The rules of algebra (T. R. Witmer, Ed. \& Trans.). New York: Dover Publications. (Original work published 1545), Dover Publications. (Original work published 1545). New York.

Domingues, J.C. (2004). Variables, limits, and infinitesimals in Portugal in the late 18th century. Historia Mathematica, 31(1), 15-33.

Euler, L. (1748). Introductio in analysin infinitorum [Introduction to the analysis of the infinite]. Lausanne: Marcum Michaelem Bousquet.

Euler, L. (1755). Institutiones calculi differentialis cum ejus usu in analysi finitorum ac doctrina serierum [Foundations of differential calculus, with applications to finite analysis and series]. Petropolitanae: Academiae imperialis scientiarum.

Farmaki, V., \& Paschos, T. (2007). Employing genetic moments in the history of mathematics in classroom activites. Educational Studies in Mathematics, 66, 83-106.

Fauvel, J. G. (1991). Using history in mathematics education. For the Learning of Mathematics, 11(2), 3-6.

Fenaroli, G., Furinghetti, F., \& Somaglia, A. (2014). Rethinking mathematical concepts with the lens of the history of mathematics: An experiment with prospective secondary teachers. Science \& Education, 23, $185-203$.

Fried, M. N. (2001). Can mathematics education and history of mathematics coexist? Science \& Education, 10(4), 391-408.

Fried, M. N. (2007). Didactics and history of mathematics: Knowledge and self-knowledge. Educational Studies in Mathematics, 66, 203-223.

Furinghetti, F., \& Radford, L. (2002). Historical conceptual developments and the teaching of mathematics: From phylogenesis and ontogenesis theory to classroom practise. In L. English (Ed.), Handbook of international research in mathematics education (pp. 631-654). Mahwah, NJ: Lawrence Erlbaum.

Furinghetti, F., \& Radford, L. (2008). Contrasts and oblique connections between historical conceptual developments and classroom learning in mathematics. In L. English (Ed.), Handbook of international research in mathematics education, 2nd ed. (pp. 626-655). New York: Routledge, Taylor and Francis.

Glaubitz, M. (2011). The use of original sources in the classroom: Empirical research findings. In E. Barbin, M. Kronfellner, \& C. Tzanakis (Eds.), Proceedings of the 6th European Summer University on the History and Epistemology in Mathematics Education (pp. 351-361). Wien: Holzhausen Verlag.

Grattan-Guinness, I. (2004a). History or heritage? An important distinction in mathematics and for mathematics education. The American Mathematical Monthly, 111(1), 1-12.

Grattan-Guinness, I. (2004b). The mathematics of the past: Distinguishing its history from our heritage. Historia Mathematica, 31(3), 163-185. 
Jahnke, H. N. (2000). The use of original sources in the mathematics classroom. In J. Fauvel, \& J. van Maanen (Eds.), History in mathematics education: The ICMI study. Dordrecht/Boston/London: Kluwer, Chapter 9.

Jankvist, U. T. (2009). A caterigorization of the "whys" and "hows" of using history in mathematics education. Educational Studies in Mathematics, 71, 235-261.

Jankvist, U. T. (2011). Anchoring students' metaperspective discussion of history in mathematics. Journal for Research in Mathematics Education, 42(4), 344-383.

Jankvist, U. T., \& Kjeldsen, T. H. (2011). New avenues for history in mathematics education: Mathematical competencies and anchoring. Science \& education, 20(9), 831-862.

Juter, K. (2006). Limits of functions as they developed through time and as students learn them today. Mathematical Thinking and Learning, 8(4), 407-431.

Kjeldsen, T. H., \& Petersen, P. H. (2014). Bridging history of the concept of function with learning of mathematics: Students' meta-discursive rules, concept formation and historical awareness. Science \& Education, 23(1), 29-45.

Lam, L. Y. (1994). Jiu zhang suanshu (Nine chapters on the mathematical art): An overview. Archive for History of Exact Sciences, 47, 1-51.

Lam, L. Y., \& Ang, T. S. (2004). Fleeting footsteps: Tracing the conception of Arithmetic and Algebra in ancient China (Rev. ed.). Singapore: World Scientific.

Lam, L. Y., \& Shen, K. (1989). Methods of solving linear equations in traditional China. Historia Mathematica, 16, 107-205.

l'Hospital, G. F. A. (1696). Analyse des infiniment petits pour l'intelligence des lignes courbes [Analysis of the infinitely small to understand curves]. Paris: (n.p.).

Moreno, L. E., \& Waldegg, G. (1991). The conceptual evolution of actual mathematical infinity. Educational Studies in Mathematics, 22, 211-231.

Mosvold, R. (2003). Genesis principles in mathematics education. In O. Bekken, \& R. Mosvold (Eds.), Study the Masters (pp. 85-96). Göteborg: Nationellt Centrum för Matematikutbildning.

Mumford, D. (2010). What's so baffling about negative numbers? - A cross-cultural comparison. In C.S. Seshadri (Ed.), Studies in the history of Indian mathematics (pp. 113-143). New Delhi: Hindustan Book Agency.

Palmgren, E. (2007). Icke-standardanalys och historiska infinitesimaler [Non-standard analysis and historical infinitesimals]. Normat, 55(4), 166-176.

Panagiotou, E. (2011). Using history to teach mathematics: The case of logarithms. Science \& Education, $20,1-35$.

Pengelley, D. (2011). Teaching with primary historical sources: Should it go mainstream? Can it? In V. Katz, \& C. Tzanakis (Eds.), Recent developments in introducing a historical dimension in mathematics education (pp. 1-8). Washington, D.C.: Mathematical Association of America.

Pengelley, D. (2012). Teaching number theory from Sophie Germain's manuscripts: A guided discovery pedagogy. Proceedings, HPM 2012 (pp. 103-113). Korea: Daejeon.

Piaget, J., \& Garcia, R. (1989). Psychogenesis and the history of science. New York: Columbia University Press.

Poincaré, H. (1899). La logique et l'intuition dans la science mathématique et dans l'enseignement [Logic and intuition in mathematical science and education]. L'Enseignement Mathématique, 1, 157-162.

Radford, L. (1997). On psychology, historical epistemology, and the teaching of mathematics: Towards a socio-cultural history of mathematics. For the Learning of Mathematics, 17(1), 26-33.

Radford, L. (2000). Historical formation and student understanding of mathematics. In J. Fauvel, \& J. van Maanen (Eds.), History in mathematics education: The ICMI study (pp. 143-170). Dordrecht: Kluwer.

Robinson, A. (1996). Non-Standard Analysis. Princeton, N.J.: Princeton University Press.

Rogers, L. (2010). History, heritage and the UK mathematics classroom. In V. Durand, S. Soury-Lavergne, \& F. Arzarello (Eds.), Proceedings of CERME 6, Working group 15: Theory and research on the role of history in mathematics education (pp. 2781-2790). Lyon, France: INRP.

Schmieden, C., \& Laugwitz, D. (1958). Eine Erweiterung der Infinitesimalrechnung [An extension of the calculus]. Mathematisches Zeitschrift, 69, 1-39.

Schubring, G. (2005). Conflicts between generalization, rigor, and intuition. New York: Springer.

Schubring, G. (2011). Conceptions for relating the evolution of mathematical concepts to mathematics learning-epistemology, history, and semiotics interacting. Educational Studies in Mathematics, 77(1), 79-104.

Sfard, A. (1991). On the dual nature of mathematical conceptions: Reflections on processes and objects as different sides of the same coin. Educational Studies in Mathematics, 22, 1-36.

Sfard, A. (1995). The development of algebra: Confronting historical and psychological perspectives. Journal of Mathematical Behavior, 14, 15-39. 
Siu, M. K. (2000). Historical support for particular subjects. In J. Fauvel, \& J. van Maanen (Eds.), History in mathematics education: The ICMI study (pp. 241-290). Dordrecht: Kluwer. Line.

Tall, D., \& Vinner, S. (1981). Concept image and concept definition in mathematics, with special reference to limits and continuity. Educational Studies in Mathematics, 12(2), 151-169.

Thomaidis, Y. (1993). Aspects of negative numbers in the early 17th century. Science and Education, 2, 6986.

Thomaidis, Y., \& Tzanakis, C. (2007). The notion of historical parallelism revisited: Historical evolution and students' conception of the order relation on the number line. Educational Studies in Mathematics, 66(2), 165-183.

Tzanakis, C., \& Arcavi, A. (2000). Integrating history of mathematics in the classroom: An analytic survey. In J. Fauvel, \& J. van Maanen (Eds.), History in mathematics education: The ICMI Study (pp. 201-240). Dordrecht: Kluwer.

Tzanakis, C., \& Thomaidis, Y. (2011). Classifying the arguments and methods to integrate history, in mathematics education: An example. In E. Barbin, M. Kronfellner, \& C. Tzanakis (Eds.), Proceedings of the 6th European summer university on the history and epistemology in mathematics education (pp. 127-136). Wien: Holzhausen Verlag.

Tzanakis, C., \& Thomaidis, Y. (2012). Classifying the arguments and methodological schemes for integrating history in mathematics education. In B. Sriraman (Ed.) Crossroads in the history of mathematics and mathematics education: The Montana mathematics enthusiast monographs (Vol. 12, pp. 247-295). Charlotte, NC: Information Age Publishing.

Unguru, S. (1975). On the need to rewrite the history of Greek mathematics. Archive for History of Exact Sciences, 15(1), 67-114.

Viirman, O., Attorps, I., \& Tossavainen, T. (2010). Different views: Some Swedish mathematics students' concept images of the function concept. Nordic Studies in Mathematics Education, 15(4), 5-24.

Wallis, J. (1685). Treatise of Algebra. London: (n.p.).

Weil, A. (1978). History of mathematics: Why and how? In O. Lehto (Ed.), Proceedings on International Congress of Mathematicians, Helsinki 1978 (Vol. 1, pp. 227-443). Helsinki: Academia Scientarum Fennicia. 